\title{
Adaptive parameter-tuning stochastic resonance based on SVD and its application in weak IF digital signal enhancement
}

\author{
Zheng Zhang ${ }^{*}$ (D) and Jinquan Ma
}

\begin{abstract}
Parameter-tuning stochastic resonance can effectively use noise to enhance signal energy, whereas its system parameters are hard to select, and how to combine it with more practical signals needs to be researched. In this study, the IF (intermediate frequency) digital signal with low SNR (signal-noise ratio) is selected as the research object, and the measuring function based on SVD (singular value decomposition) that is not dependent on prior knowledge is proposed as the evaluation function to optimize the parameters of stochastic resonance system. The nature of the stochastic resonance is first described from the eigenspace of the signal. After the analysis of the effects of different system parameters, amplitude normalization is employed to optimize only one parameter, simplifying the algorithm. Finally, an adaptive parameter-tuning stochastic resonance method based on AFSA (artificial fish swarm algorithm) is developed for three types of modulated signals, achieving an optimum matching of noisy signals and non-linear systems at fast convergence speed. According to the simulation, the proposed algorithm is proven effective, efficient, and robust, laying a solid foundation for the subsequent signal processing work.
\end{abstract}

Keywords: Stochastic resonance, SVD, Evaluation function, Weak signal processing, Adaptive optimization algorithm

\section{Introduction}

In the non-cooperative communication system as shown in Fig. 1, the received digital signals are often weak and at low SNR. Given that the prior knowledge of the transmitted signals cannot be gained in advance, if the information contained in the signals is to be acquired, the blind signal processing technique must be used to estimate the necessary parameters (e.g., carrier frequency, symbol rate, modulation mode) before demodulation and information recovery. Different parameter estimation or modulation recognition algorithms will bring different performances, whereas the quality of the signal, as an important factor, will undoubtedly have huge impacts on the algorithm results. Scholars have performed a lot of research on signal processing at low SNR. The high-order spectrum method [1-3] can suppress the

\footnotetext{
* Correspondence: zz2018zheng@gmail.com

Information Engineering University, Zhengzhou 450001, People's Republic of China
}

additive colored Gaussian noise of unknown power spectrum. The wavelet transform technique $[4,5]$ is considered suitable for the noise removal of transient signals and can inhibit the interference of high-frequency noise. The method of calculating high-order cumulant [6] is insensitive to Gaussian noise. The cyclic spectrum $[7,8]$ has certain anti-noise performance, etc. These methods primarily focus on suppressing noise to the greatest extent. In recent years, however, with the in-depth study of non-linear dynamics and statistical physics theory, stochastic resonance (SR) method has been developed [9] using a non-linear system to convert part of the energy of the noise into signal, thereby enhancing the signal and reducing the noise.

The initial study of SR was limited to periodic signals. Later, the aperiodic stochastic resonance (ASR) proposed by Collins et al. [10] and the parameter-tuning stochastic resonance (PSR) proposed by Xu et al. [11] were broadly used. On the one hand, ASR breaks through the 


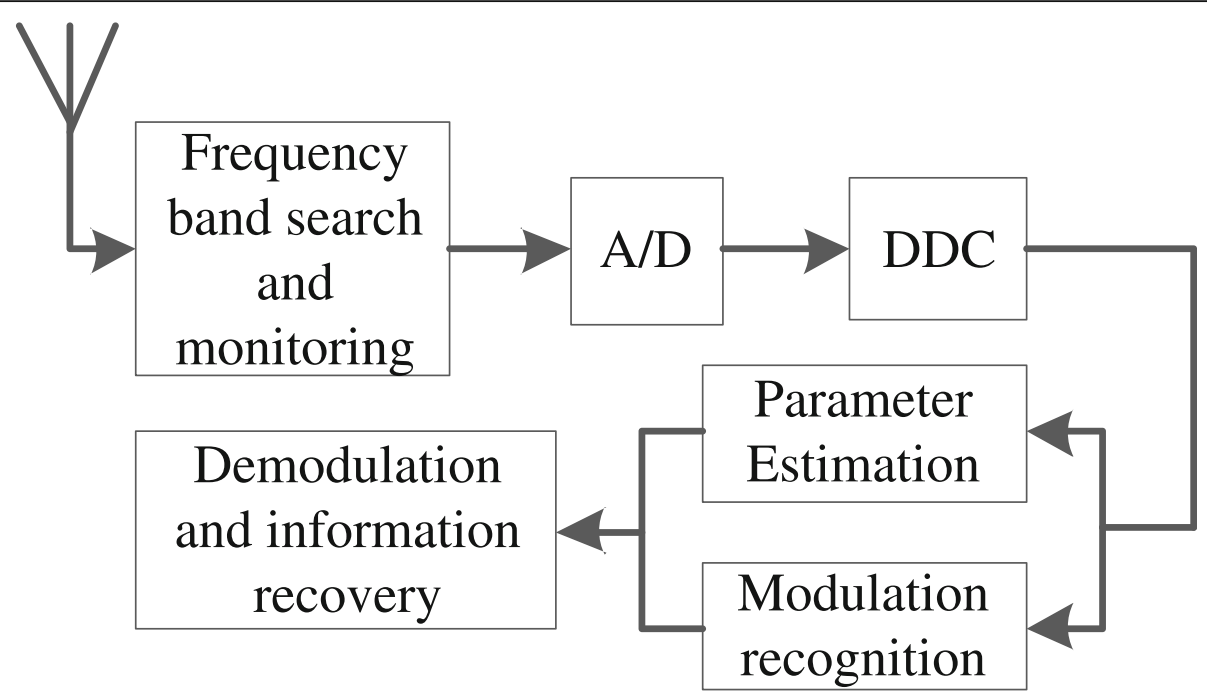

Fig. 1 Signal processing steps under non-cooperative communication condition

conditional constraints of the input signals, demonstrating that aperiodic signals can also stimulate stochastic resonance system. On the other hand, PSR solves the problem that only the system parameters need to be adjusted without adjusting the noise can achieve the best output. Stochastic resonance is applied in a large number of fields: medicine [12], electromagnetics [13, 14], mechanical fault detection $[15,16]$, signal processing [17], etc. In the field of digital signal processing, Duan and Abbott [18] explored the detectability of the SR bistable receiver for detecting binary modulated signals. Sun and Lei [19] studied the use of ASR processor to detect the pulse amplitude modulation (PAM) signals and applied it to the digital watermark. Dalabaev et al. [20] studied the application of PSR in baseband digital signals. Liu et al. [21] proposed a PSR receiver to improve the reception performance of PAM signals. Liang et al. [22] derived the expression of the bit error rate of the bistable stochastic resonance system under the coherent receiver. Zhan and Duan [23] applied PSR to the parameter estimation of the signals. But all these studies are carried out under the fixed stochastic resonance system parameter and the fixed type of signal.

For adaptive PSR, the choice of system parameters plays a critical part in the performance of the output. This is because only when the signal, noise, and system are optimally matched, can the noise be weakened, and can the target signal be enhanced to the greatest extent. Thus, Tong et al. [24] studied the adaptive stochastic resonance method of PSO (particle swarm optimization) and analyzed the parameters that affect system stability using the scale-transform stochastic resonance solution procedure. The adaptive stochastic resonance method based on artificial fish swarm algorithm (AFSA) $[25,26]$ was studied only for sinusoidal signal and cannot be applied to more complex aperiodic signals, which limited its application [27, 28]. The AFSA is an effective optimization algorithm, characterized by parallelism, simplicity, and fastness. However, how to select the best system parameters for various IF digital signals needs further studies.

In this work, a weak IF digital signal enhancement method based on adaptive parameter-tuning stochastic resonance is proposed. Unlike the traditional stochastic resonance, the evaluation function based on SVD combined with AFSA is used to make the weak IF signal tend to achieve the best enhancement by stochastic resonance with the most optimal system parameters. The proposed method has a wider scope of application, which can be used for three types of modulated signals and is more suitable for engineering practice.

The rest of the paper is organized as follows. In Section 2, the definition of IF digital signals, the stochastic resonance theory, and AFSA algorithm are explained. In Section 3, the method scheme is described, including SVD evaluation function, system parameter analysis, and module framework. In Section 4, the simulation results and analysis are described. Finally, conclusions are given in Section 5.

\section{Background knowledge \\ 2.1 IF digital signal}

IF digital signal refers to the digital signal whose carrier frequency is maintained at a predetermined value which is between baseband and radio frequency (RF) after down-conversion processing. There are three types of IF signals studied in this study: 
(1) Multiple amplitude shift keying (MASK) directly maps the information of the symbol to the carrier amplitude, and its time domain expression is:

$$
\operatorname{sMASK}(t)=\left[A \sum_{n} \operatorname{ang}(t-n T s)\right] \cos (2 \pi f \mathrm{c} t+\theta)
$$

where $a n \in\{2 m-1-\sqrt{M} \mid m=1,2, \ldots, M\}$.

(2) Multiple phase shift keying (MPSK) uses the phase change of carrier to transmit digital information, and its time domain expression is:

$$
s \operatorname{MPSK}(t)=\left[A \sum_{n} g(t-n T s)\right] \cos (2 \pi f c t+\theta+\phi n)
$$

where $\phi_{n} \in\{(2 m-1) \pi / M \mid m=1,2, \ldots, M\}$.

(3) Multiple quadrature amplitude modulation (MQAM) is a method of mixed amplitude and phase modulation whose time domain expression is:

$$
\begin{aligned}
s \operatorname{MQAM}(t) & =\left[A \sum_{n} a n g(t-n T s)\right] \cos (2 \pi f \mathrm{c} t+\theta) \\
& +\left[A \sum_{n} b n g(t-n T s)\right] \sin (2 \pi f \mathrm{c} t+\theta)
\end{aligned}
$$

where $a n, b n \in\{2 m-1-\sqrt{M} \mid m=1,2, \ldots, M\}$.

In (1), (2), and (3), $A$ is the amplitude, $g(t)$ is the pulse shaping function and only rectangular shaping is considered, $T s$ is the symbol duration, $f_{c}$ is the carrier frequency, $\theta$ is the initial phase of the carrier, and $M$ is the symbol mapping number(e.g., $M=2,4,8 \ldots)$.

\subsection{Bistable system and adiabatic approximation theory}

The bistable system is a typical type of non-linear system that can be represented by the Langevin equation [29]:

$$
\frac{d x}{d t}=a x-b x^{3}+s(t)+\Gamma(t)
$$

where $a$ and $b$ are the parameters of the system, $s(t)$ is the input signal, and $\Gamma(t)$ is the Gaussian white noise with mean value of 0 and intensity of $D$, conforming to
$\langle\Gamma(t)\rangle=0,\langle\Gamma(t) \Gamma(0)\rangle=2 D \delta(t)$ where $\langle\cdot\rangle$ represents the time average.

IF digital signal, as the input, can be regarded as the single frequency signal with the fixed amplitude and phase in any non-zero symbol interval, which can be expressed by the following equation:

$$
s(t)=A \cos (2 \pi f c t+\phi)
$$

where $k T s \leq t \leq(k+1) T s, k=1,2, \ldots, N$, and $N$ is the number of symbols.

In this way, the aperiodic IF digital signal can be analyzed as the periodic signal within a symbol interval. Therefore, it can be treated as a global aperiodic signal for PSR processing and local periodic signal for analyzing.

The potential function corresponding to (4) is:

$$
\begin{aligned}
U(x)= & -\frac{1}{2} a x^{2}+\frac{1}{4} b x^{4} \\
& -x(A \cos (2 \pi f c t+\phi)+\Gamma(t))
\end{aligned}
$$

Equation (6) describes the particle's overdamped motion in a double potential well driven by external force and noise simultaneously. When there is no external force and noise, the potential function curve is shown in Fig. 2.

It can be seen that the two potential wells $x 1,2= \pm$ $\sqrt{a / b}$ are symmetric about the zero point, and the barrier height is $\Delta U=a^{2} / 4 b$. When the system is excited by only external force, as long as $A$ is less than the critical value $A_{\mathrm{c}}\left(A c=\sqrt{4 a^{3} / 27 b}\right)$, the particle can only perform the local periodic motion in a certain potential well. When there is only noise, the particle switches between the two potential wells according to Kramers' transition rate $r_{\mathrm{k}}$, which is expressed as $r_{\mathrm{k}}=\frac{a}{\sqrt{2} \pi} \exp \left(-\frac{\Delta U}{D}\right)$. When the sys-

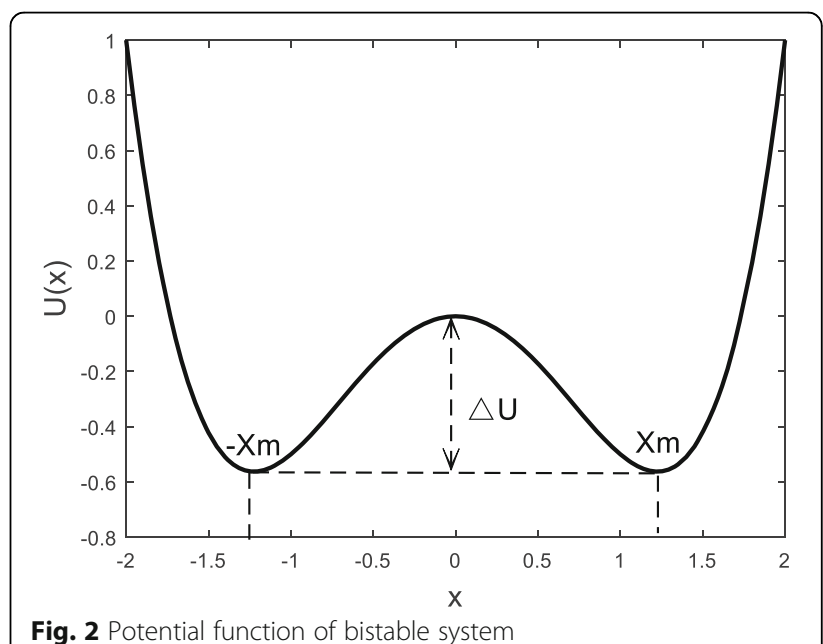


tem is excited by both signal and noise, the particle can exceed the barrier with the help of noise even if $A<A c$ and periodically flips in the two potential wells according to the signal frequency. Since the energy of the overstepping barrier is much larger than the energy of the signal itself, the periodic characteristic of the signal within one symbol interval is amplified, which means the stochastic resonance phenomenon occurs. The probability distribution function $\rho(x, t)$ of the variable $x$ follows the Fokker-Planck equation:

$$
\begin{aligned}
\frac{\partial \rho(x, t)}{\partial t}= & -\frac{\partial}{\partial x}\left[\left(a x-b x^{3}+A \cos (2 \pi f c t)\right) \rho(x, t)\right] \\
& +D \frac{\partial^{2}}{\partial x^{2}} \rho(x, t)
\end{aligned}
$$

where the initial condition is $\rho\left(x, t_{0} \mid x_{0}, t_{0}\right)=\delta\left(x-x_{0}\right)$.

When $f c$ of the signal is small enough and the amplitude and noise intensity are much less than 1 at the same time, it can be considered that the time taken for the system to reach the equilibrium state in potential wells is much smaller than the time taken for the overall equilibrium of the probability between two potential wells, and is also much smaller than the time taken by the system to change along with the input signal, which is the adiabatic approximation [30,31].

After analytic derivation [32], the power spectrum of the system output can be obtained:

$$
\begin{aligned}
G(\omega)= & G_{N}(\omega)+G_{S}(\omega) \\
= & \left(1-\frac{r_{\mathrm{k}}^{2} A^{2} x_{\mathrm{m}}{ }^{2}}{2 D^{2}\left(r_{\mathrm{k}}{ }^{2}+\pi^{2} f \mathrm{c}^{2}\right)}\right) \frac{r_{k} x_{\mathrm{m}}{ }^{2}}{r_{\mathrm{k}}{ }^{2}+\pi^{2} f \mathrm{c}^{2}} \\
& +\frac{\pi A^{2} x_{\mathrm{m}}{ }^{4} r_{\mathrm{k}}{ }^{2}}{2 D^{2}\left(r_{\mathrm{k}}{ }^{2}+\pi^{2} f \mathrm{c}^{2}\right)}[\delta(\omega-\omega c)+\delta(\omega+\omega c)]
\end{aligned}
$$

where $\omega_{\mathrm{c}}=2 \pi f \mathrm{c}$ and $x_{m}=\sqrt{a / b}$.

Equation (8) reflects the energy conversion result which consists of two parts: noise energy and signal energy. During the stochastic resonance process, the signal power spectrum appears amplified peak value, and the total output power of the system is maintained as $2 \pi x_{\mathrm{m}}{ }^{2}$ with no change.

In order to overcome the limitation of adiabatic approximation theory that the input must satisfy small parameters, the following variables are introduced to normalize the input [33]:

$$
z=x \sqrt{\frac{b}{a}}, \tau=a t
$$

So, we can get the final expression as follows:

$$
\begin{aligned}
\frac{d z}{d \tau}= & z-z^{3}+\sqrt{\frac{b}{a^{3}}} A \cos \left(\frac{2 \pi f \mathrm{c}}{a} \tau+\phi\right) \\
& +\sqrt{\frac{2 D b}{a^{3}}} \xi(\tau)
\end{aligned}
$$

The normalization compresses the signal frequency and scales the amplitude of the signal and the noise so that the stochastic resonance can be applied to more actual signals.

\subsection{Artificial fish swarm algorithm}

Artificial fish (AF) is the virtual entity of the real fish, which simulates four instinctive behaviors of fish: preying, swarming, following, and moving to survive in the surrounding environment. Through each evaluation, it selects a current optimal behavior to carry out, obtaining higher food concentration.

As shown in Fig. 3, the current state of an $\mathrm{AF}$ is $X$, Visual is its visual distance, and the state $X V$ is the position in Visual at a certain moment. The AF compares the $X$ with the $X V$, thereby deciding to move forward or continue to patrol other locations within the Visual. The greater the number of patrols, the more comprehensive the understanding about the states of the Visual, which helps to make correspondingly intelligent judgments and decisions to maintain colonies. Of course, it is not necessary to traverse the environment with multiple or infinite states, which allows AF to have local optimization with some uncertainty and it is helpful for finding the global optimum.

The variable part includes $M$ (number of AF), GEN (number of reproductions), $\mathbf{X}$ (state of the AF, which

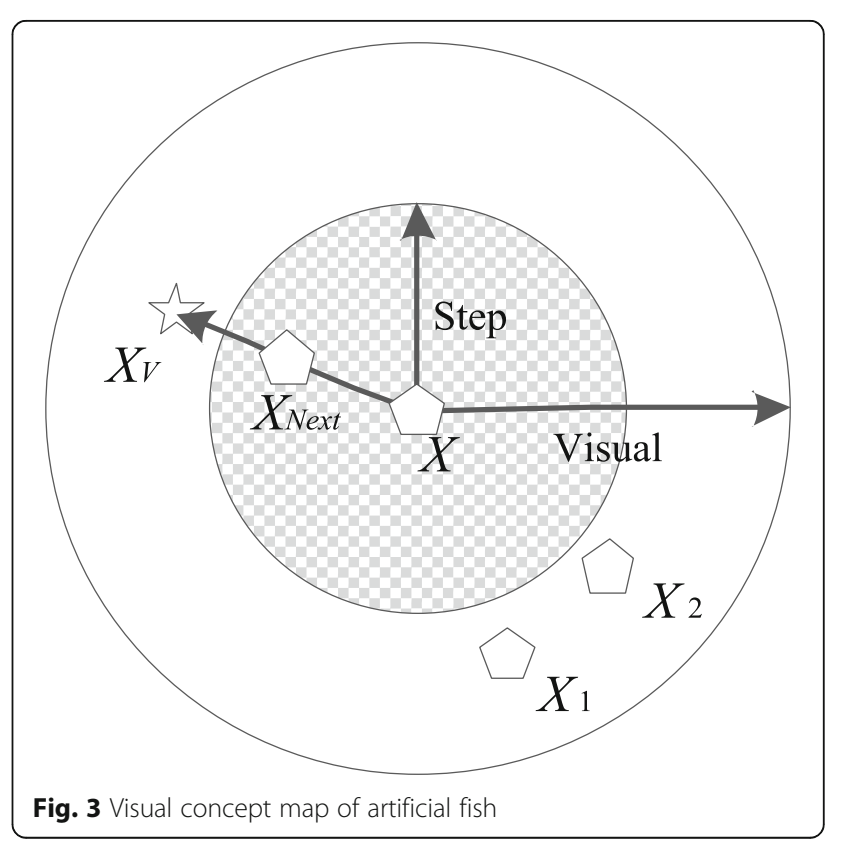


is a vector of the variable to be optimized), Step (maximum step size of movement), Visual (visual distance of $\mathrm{AF}$ ), $T$ (maximum number of attempts), $\delta$ (crowd factor), $d i j=|\mathbf{X} i-\mathbf{X} j|$ (distance between $\mathrm{AF}$ individuals $i$ and $j$ ), and $Y$ (food concentration).

AF reaches the highest concentration of food through the following four behaviors.

1. Prey: Suppose the artificial fish $i$ has a current state $\mathbf{X} i$ and selects a state $\mathbf{X} j$ within its Visual randomly:

$$
\mathbf{X} \boldsymbol{j}=\mathbf{X} i+\text { Visual } \cdot \operatorname{Rand}()
$$

If $Y j<Y i$, then goes forward a step in that direction; otherwise, if the condition is still not satisfied after $T$ times, it moves a step randomly.

$$
\mathbf{X}_{i}^{t+1}=\mathbf{X}_{i}^{t}+\frac{\mathbf{X}_{j}-\mathbf{X}_{i}^{t}}{\left\|\mathbf{X}_{j}-\mathbf{X}_{i}^{t}\right\|} \cdot \text { Step } \cdot \operatorname{Rand}()
$$

2. Swarm: Suppose $n f$ is the number of its partners in the current neighborhood and $\mathbf{X} c$ is the center position of them, if $Y c / n f>\delta Y i$, then moves one step towards $\mathbf{X} c$, otherwise executes the preying.

3. Follow: Let $\mathbf{X} j$ has the maximum value $Y j$ among partners, if $Y j / n f>\delta Y i$, then moves one step towards $\mathbf{X} \boldsymbol{j}$, otherwise preys.

4. Move: Default behavior of preying.

\section{Methods}

Since the signal is received without any prior knowledge, it is not possible to be processed only in one symbol duration. The target signal contains a large unknown number of symbols, so it is aperiodic with a certain bandwidth. Therefore, the stochastic resonance result cannot be measured by the signal-to-noise ratio of the single-frequency signal, which is defined as the ratio of the amplitude at the signal frequency in the power spectrum of the output signal to the same-frequency background noise [32]. Later, scholars proposed the cross-correlation function as a measurement for aperiodic signals [34], but it is based on the simple waveform matching of input and output signals. There is also a measurement of bit error rate for digital signals [35], but in fact, it cannot be measured based on the received signals without knowing the correct sequence of symbols. Therefore, we need a measuring function that does not require any prior knowledge.

In this section, singular value decomposition (SVD) is used to measure the effect of the stochastic resonance output and is combined with AFSA as evaluation function to find the optimal system parameters for weak IF digital signals.

\subsection{Evaluation function based on SVD}

There is an important parameter in AFSA: food concentration $Y$, which is the evaluation function in the optimization process.

SVD can be used to estimate the signal-to-noise ratio in digital communications [36]. As a measuring function of ASR, it does not need accurate estimation and is just a relative quantity. In other words, the value of the function itself is not important, and it is significant that it can vary with the system parameters and reach the peak at the optimum situation.

It is assumed that the signal $s(t)$ passes through the additive white Gaussian noise channel and is expressed as $y(n)=s(n)+w(n)$ after sampling, where $w(n)$ is the noise with zero mean and $\sigma_{w}^{2}$ variance. The distributions of the signal and noise are independent. The autocorrelation matrix of the signal is:

$$
\begin{aligned}
\mathbf{R} y y & =E\left[y(n) y^{\mathrm{H}}(n)\right] \\
& =E\left\{[s(n)+w(n)][s(n)+w(n)]^{\mathrm{H}}\right\} \\
& =E\left\{\left[s(n) s^{\mathrm{H}}(n)\right]+E\left\{\left[w(n) w^{\mathrm{H}}(n)\right]\right.\right. \\
& =\mathbf{R} s s+\mathbf{R} w w
\end{aligned}
$$

Since the matrices $\mathbf{R} y y$, $\mathbf{R} s s$, and $\mathbf{R} w w$ in (13) are symmetric, SVD can be diagonalized as:

$$
\begin{aligned}
\mathbf{R} y y & =\mathbf{R} s s+\mathbf{R} w w=\mathbf{V} \boldsymbol{\Lambda} y \mathbf{V}^{\mathrm{H}} \\
& =\mathbf{V}(\boldsymbol{\Lambda} s+\boldsymbol{\Lambda} w) \mathbf{V}^{\mathrm{H}}
\end{aligned}
$$

where $\mathbf{V}$ is an orthogonal matrix and

$$
\begin{gathered}
\boldsymbol{\Lambda} s=\operatorname{diag}(\lambda 1, \cdots, \lambda p, 0, \cdots 0) m \times m \\
\boldsymbol{\Lambda} w=\operatorname{diag}\left(\sigma_{w}^{2}, \sigma_{w}^{2}, \cdots \sigma_{w}^{2}\right) m \times m \\
\boldsymbol{\Lambda} y=\operatorname{diag}\left(\lambda 1+\sigma_{w}^{2}, \lambda 2+\sigma_{w}^{2}, \cdots,\right. \\
\left.\lambda p+\sigma_{w}^{2}, \sigma_{w}^{2}, \cdots \sigma_{w}^{2}\right) m \times m \\
(\lambda 1>\lambda 2>\cdots>\lambda p)
\end{gathered}
$$

for which $m$ is the order and $\lambda i, i=1,2, \ldots p$ are singular values.

Taking the BPSK signal as an example, we use two different sets of stochastic resonance system parameters including a and $b$ to process it, and then perform SVD with an order of 10 on the original signal and two output signals to observe the effect of stochastic resonance from the perspective of the eigenspace. The singular values of all signals are compared from large to small, as shown in Fig. 4.

For the original signal, $\lambda 1=0.349, \lambda 2=0.111, \lambda 3=$ $0.043, \ldots, \lambda 10=0.037 ;$ after stochastic resonance 
processing under the first set of system parameters (hereafter called SR output1), $\lambda_{1}^{\prime}=1.095, \lambda_{2}^{\prime}=0.280, \lambda_{3}^{\prime}=0.02$ $8, \ldots, \lambda_{10}^{\prime}=0.001$, and under the second set of system parameters (hereafter called SR output 2 ), $\lambda_{1}^{\prime \prime}=0.580, \lambda_{2}^{\prime \prime}=0$. $216, \lambda_{3}^{\prime \prime}=0.061, \ldots, \lambda_{10}^{\prime \prime}=0.003$. Comparing the original signal with the SR output 1, we can conclude that the stochastic resonance causes the first three singular values' increase and the latter seven values' decrease, which proves that the enhancement of signal energy is related to the variation of the singular values. Furthermore, comparing the SR output 1 with the SR output 2 , we can infer that the degree of the singular value change varies with the different system parameters. In addition, it shows that the stochastic resonance can indeed use the noise energy to enhance the signal, and at the same time, the noise energy is suppressed, which demonstrates some of the noise energy is transferred to signal after processing. To sum up, this experiment proves that it is feasible to find the optimal system parameters to achieve the maximum energy conversion by using SVD.

In the application of SNR estimation, the minimum description distance (MDL) [37] is needed to determine the dimension of the signal space. However, the measuring function represents the relative meaning rather than the absolute, so it is unnecessary to determine the optimal dimension $p$. There are two advantages: one is the invalidation of the MDL in the case of weak signals is avoided and the other is the simplicity of the algorithm is guaranteed. $p$ is fixed to 3 in this paper; thus, we define the measuring function (SRIF stands for stochastic resonance of IF digital signals), which is expressed by:

$$
Y \text { SRIF }=10 \times \log \frac{\sum_{k=1}^{p}\left(\lambda k-\sigma_{w}^{2}\right)}{m \times \sigma_{w}^{2}}
$$

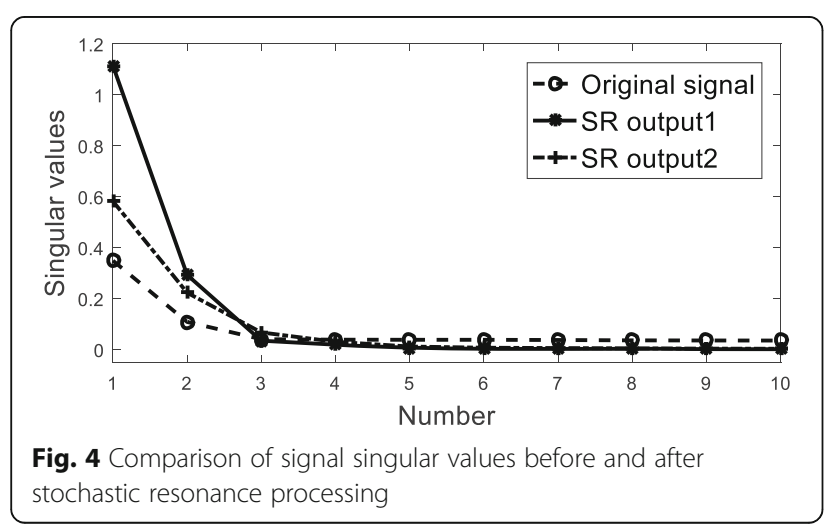

\subsection{Analysis of the parameters of the stochastic resonance system}

Research shows that the system parameters play a crucial part in Kramers' transition rate $r_{\mathrm{k}}$ of the system. The adjustment of the parameter $a$ can adapt to the input signals with different changing speeds. It is known from (10) that the normalization stretches the signal for a times in time domain, which is also equivalent to $1 / a$ time compression in the frequency domain. Also, the amplitude scale of the input is normalized by $\sqrt{b / a^{3}}$. Above conclusions demonstrate that the parameter a mainly determines whether stochastic resonance can occur, and both $a$ and $b$ affect the amplitude. In order to prove the effect of different system parameters, we take BPSK as an example, as shown in Fig. 5.

As shown in Fig. 5 , when $a=b=f$ c, the BPSK signal is enhanced by stochastic resonance to some extent; however, when $a=50 f c, b=f c$, waveform distortion occurs in the output signal, which proves that the parameter $a$ affects the quality of the output. On the contrary, if $a=$ $f c, b=50 f c$, the enhancement level of the output does not change, but the amplitude is increased by about 10 times, which proves that the parameter $b$ mainly affects the amplitude of the output. According to this feature, the influence of the parameter $b$ can be neglected by normalizing the amplitude of the stochastic resonance output, so that the complexity of the optimization can be reduced by half. In the optimization process, $b$ and $a$ remain the same size specifically.

The previously defined SVD-based measuring function YSRIF is used as the evaluation function in parameter optimization. As shown in Fig. 6, YSRIF has an optimal value within a certain range of the parameter $a$, which means that the system and the signal and noise are optimally matched and the noise triggers the maximum transfer of power to the signal in the system with optimal parameters.

\subsection{Module framework}

Design the stochastic resonance module as Fig. 7.

Step 1: Normalize the amplitude of the input to prevent the amplitude from being too large or too small.

Step 2: Determine the system parameters. Make $b=a$ and $h=1 / f s$, which is the iteration step for step 3. How to choose $a$ adaptively will be discussed later.

Step 3: Use the fourth-order Runge-Kutta method [33] to iterate and calculate the output.

Step 4: Pass the signal through the moving average filter. As shown in Fig. 8, the direct output signal has an amplitude drift phenomenon in which the amplitude of the symbol is unstable. The moving average filter can be expressed as: 

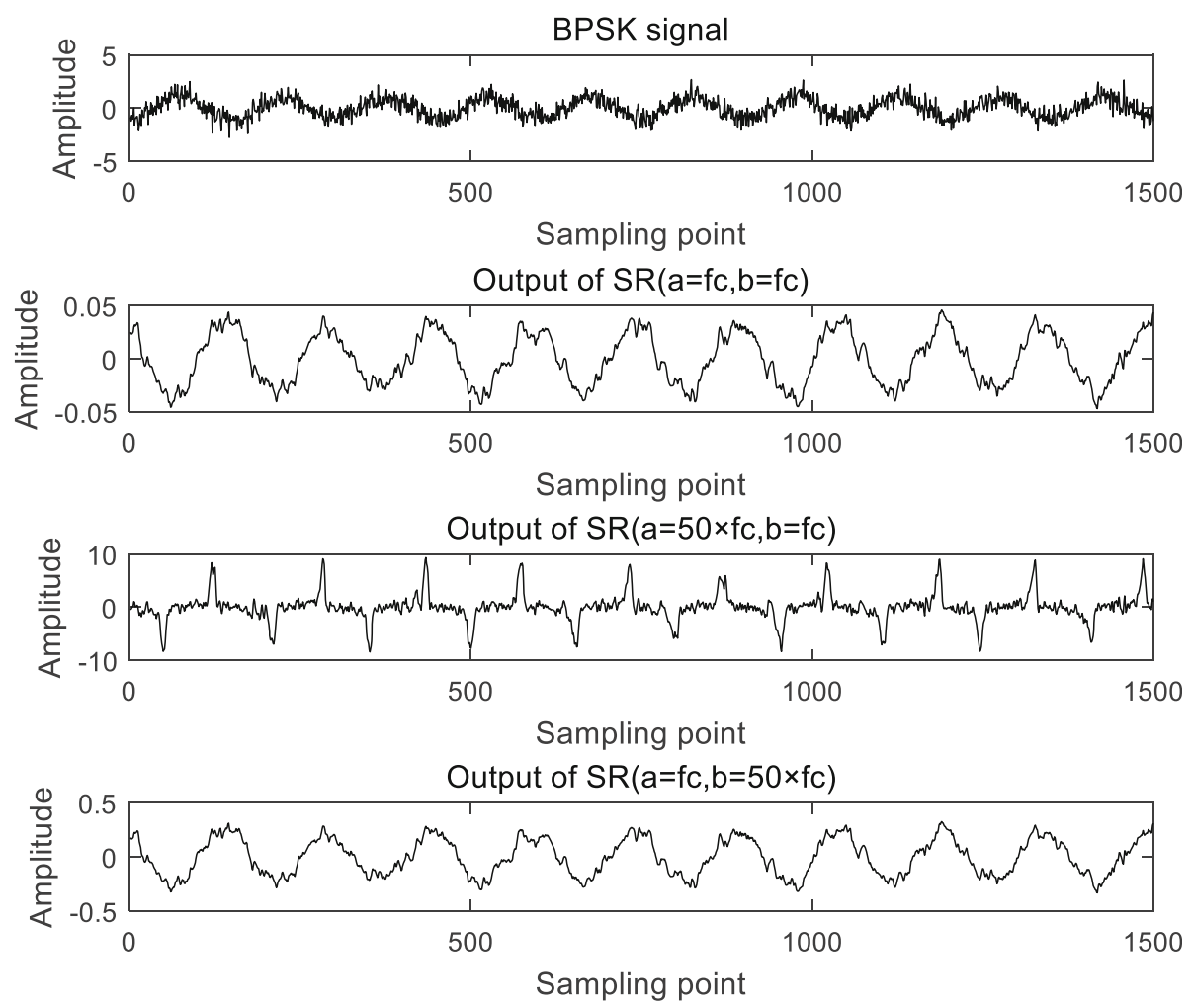

Fig. 5 Effect of different system parameters on the stochastic resonance output

$$
S^{\prime}(k)=S(k)-\frac{1}{2 K+1} \sum_{i=-K}^{K} S(k+i)
$$

where $S$ and $S$ represent the signals before and after the filter, respectively, and $K$ is the sliding length.

Step 5: Normalize the amplitude of the output to remove the influence of the parameter $b$, and finally obtain the processed signal.

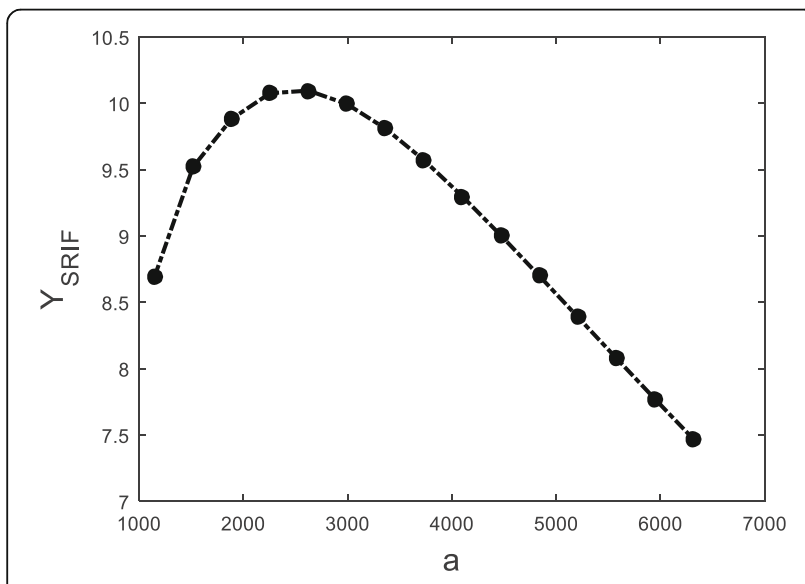

Fig. 6 Effect of $a$ on YSRIF
The stochastic resonance module is embedded into the whole algorithm framework which is shown in Fig. 9 to complete the weak IF digital signal enhancement based on AFSA.

The overall structure is divided into three parts, namely the input module, the AFSA module, and the output module.

(1) Input module: The received signal is usually the RF signal. It is sampled with being roughly measured the carrier frequency, then moved to the intermediate frequency, and intercepted as the input of the next module. The specific value of the intermediate frequency can be unknown, except for the order of magnitude, e.g., $1 \mathrm{~K} \mathrm{~Hz}, 10 \mathrm{M} \mathrm{Hz}$, etc.

(2) The AFSA module is the core part, and the specific steps of which are as follows:

First, initialize the parameters. Assuming the IF is on the order of $1 \times 10^{m} \mathrm{~Hz}$, the initial optimization range of $a$ is set as $\left[1 \times 10^{m-1}, 1 \times 10^{m+1}\right]$, the Visual is set as $0.5 \times$ $10^{m-1}$, and the settings of the remaining parameters $T, \delta$, $\boldsymbol{G E N}$, and $M$ can be properly set regardless of the magnitude order. 


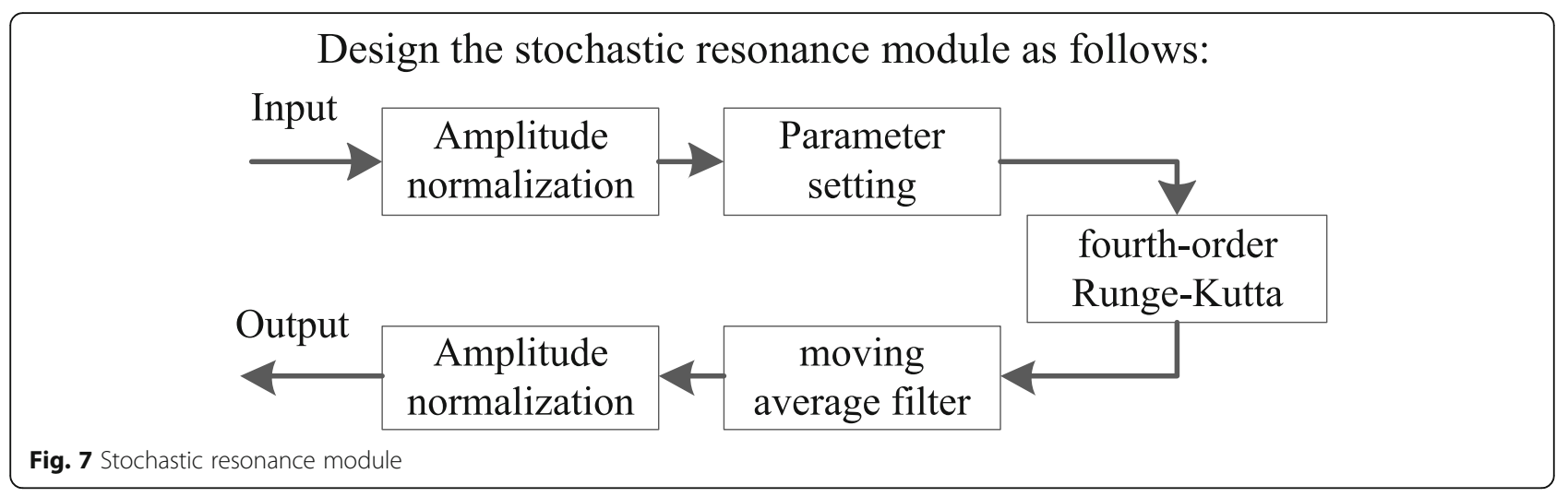

Second, the stochastic resonance module is used to calculate the food concentration, and YSRIF is used to evaluate and select the current optimal parameter.

Third, AF performs behavior functions sequentially, iterated, updated, and recorded generation after generation;

At last, when the number of iterations reaches the preset value, the current optimal parameter is the output.

(3) Output module: The received signal is passed through the stochastic resonance module under the optimal parameters to obtain the enhanced signal.

\section{Results and discussion}

4.1 Experiment 1: Effectiveness validation

First, the IF digital signals are simulated, including seven kinds: 2ASK, 4ASK, BPSK, QPSK, 8PSK, 16QAM, and 64QAM. The parameters of the signals are uniformly set as amplitude $A=1$, symbol rate $R \mathrm{~s}=1000 \mathrm{Bd}$, carrier frequency $f_{\mathrm{c}}=1 \times 10^{4} \mathrm{~Hz}$, number of symbols $N=20$, sampling rate $f_{\mathrm{s}}=1 \times 10^{6} \mathrm{~Hz}$, and $\mathrm{SNR}=0 \mathrm{~dB}$. The AFSA parameters are set as $M=50, \mathrm{GEN}=10, T=20$, Visual = 1000 , Step $=500$, and $\delta=0.5$, and the initial optimization range is $\left[1 \times 10^{3}, 1 \times 10^{5}\right]$.

The AFSA module is used to acquire the optimal system parameter, and the optimal values and the maximum food
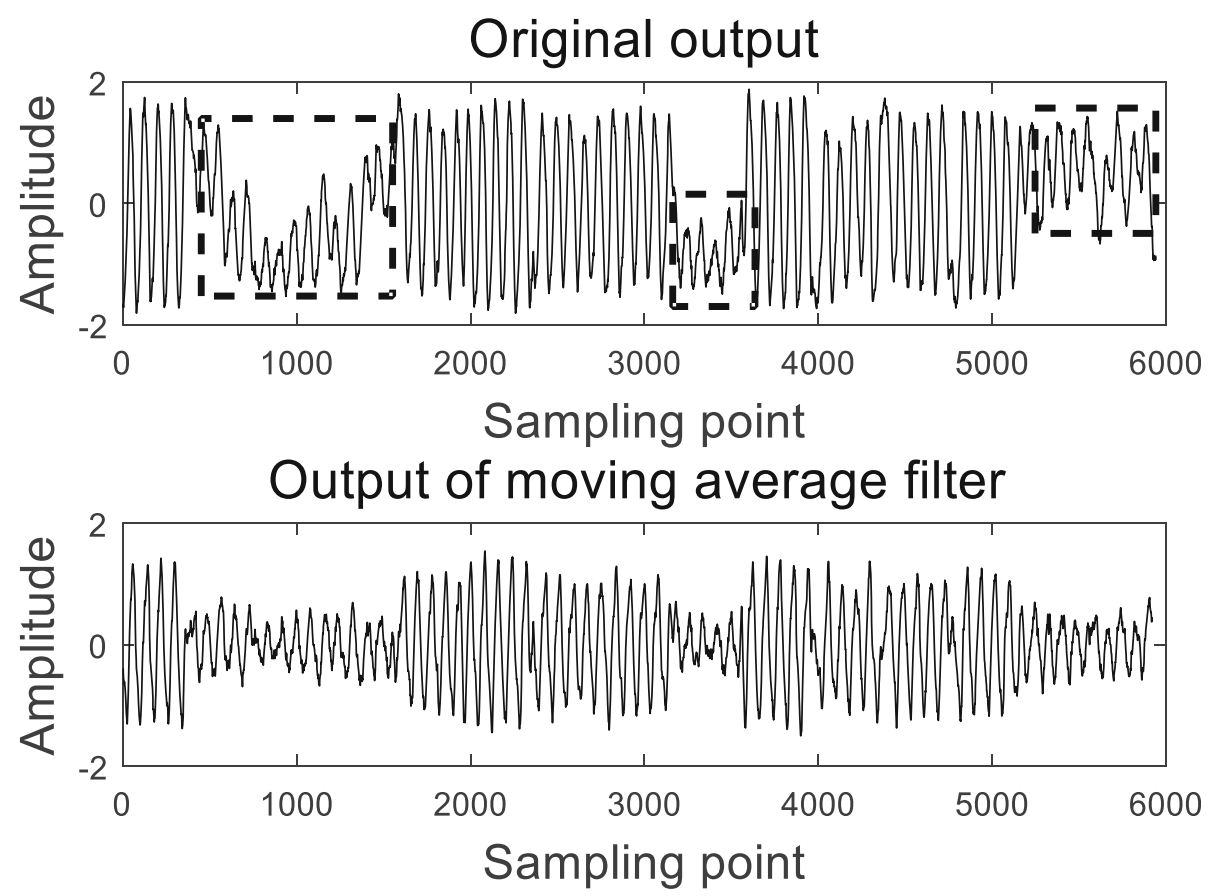

Fig. 8 Comparison before and after moving average filtering 


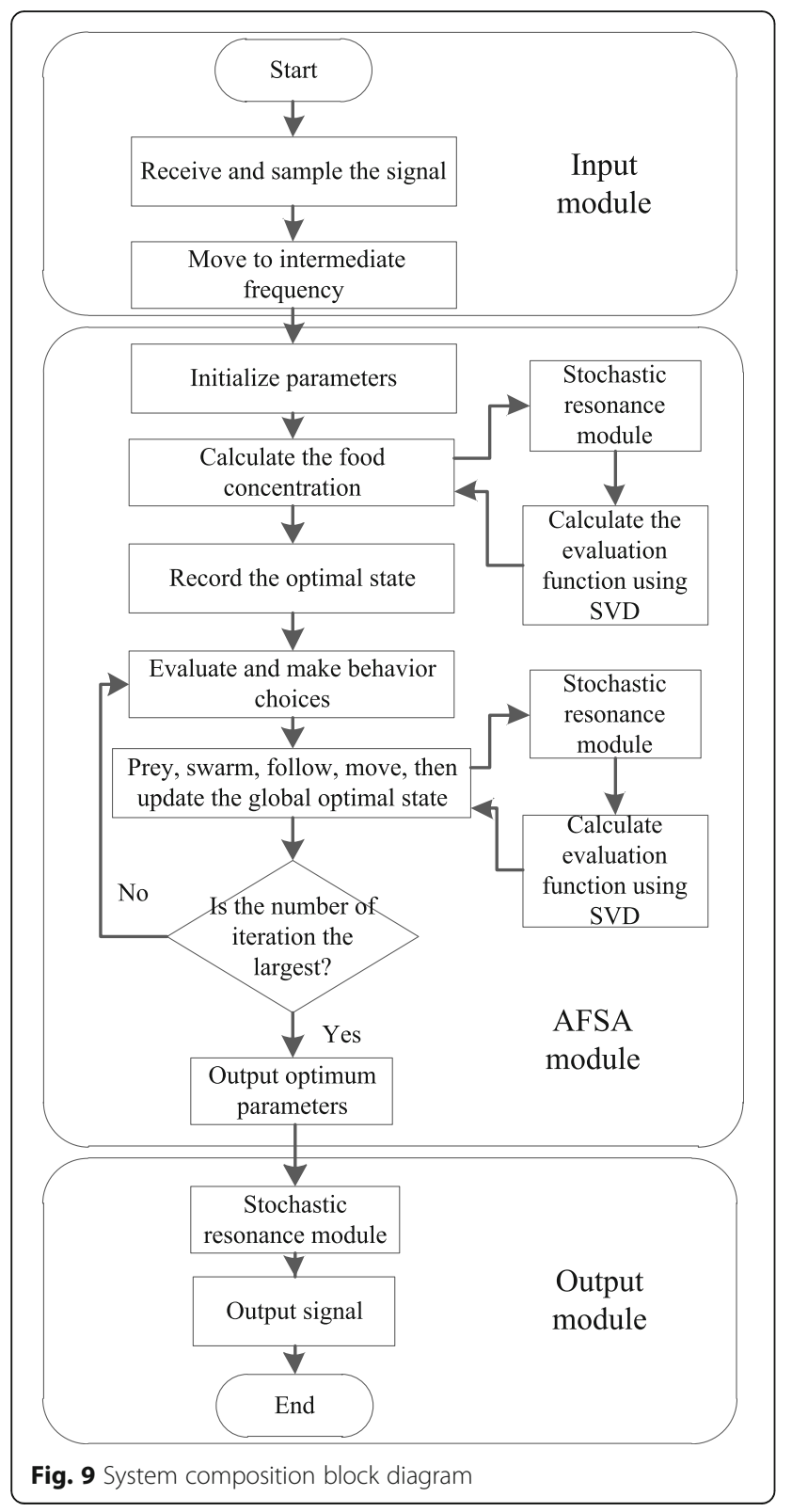

concentration are recorded with the increasement of the iteration number, as shown in Fig. 10.

Taking the convergence curve of the 64QAM as an example, the optimum parameter of the first AF generation is about $1.45 \times 10^{4}$ with the food concentration of 3.408, indicating that the food concentration under this value is the highest among these $50 \mathrm{AFs}$ in the initial searching stage. Next, the second generation continues to go ahead in a large step, and the food concentration reaches 6.641. In the third generation, it is close to arrive at the optimal parameter position with the highest food concentration with a small step. Finally, it reaches the optimal position in the fifth generation. The curves illustrate that in the early reproduction stage, the AF can optimize

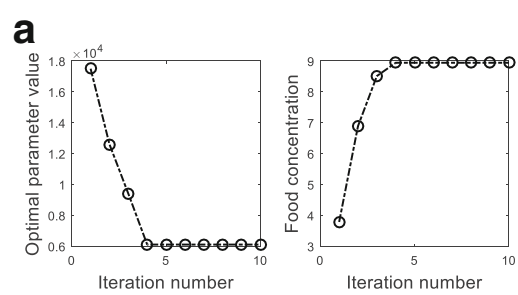

b
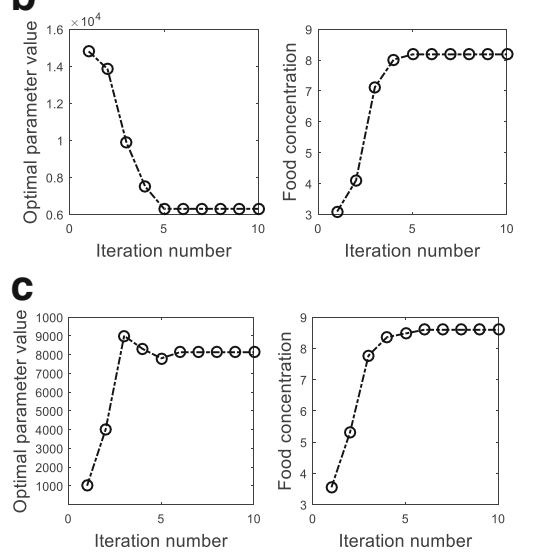

d

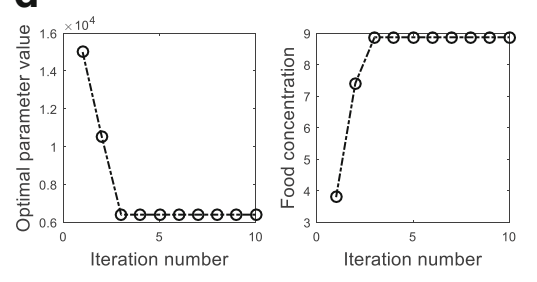

e

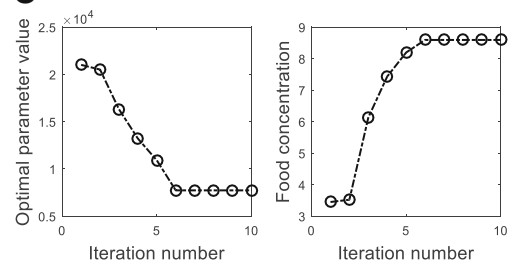

f
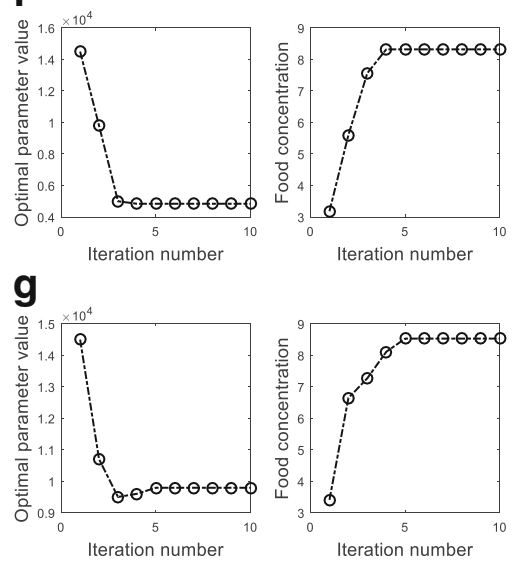

Fig. 10 Convergence curve. a 2ASK. b 4ASK. c BPSK. d QPSK. e 8PSK. f 16QAM. g 64QAM 


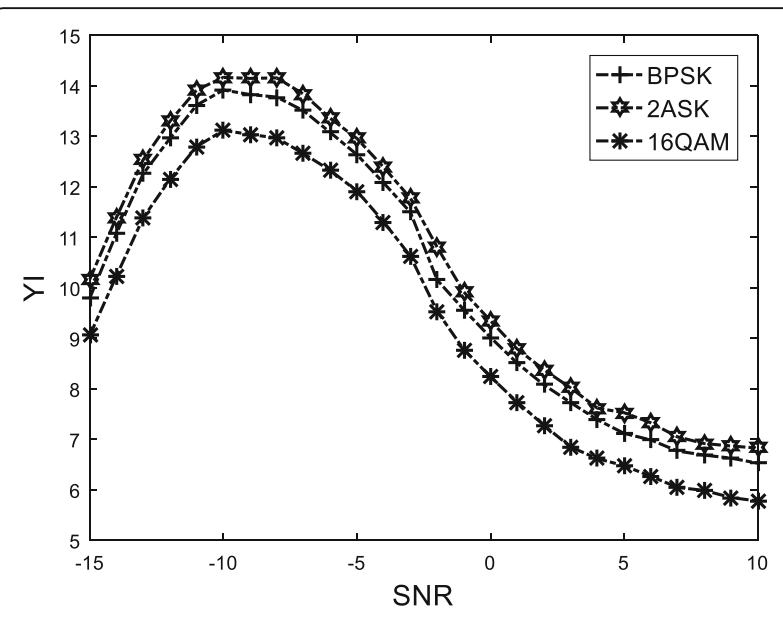

Fig. 11 Signal enhancement result after PSR. a 2ASK. b BPSK. c 16QAM

with a fast speed and approach the optimal position gradually with a small step in the later stage, thus achieving an ideal convergence result. The results manifest that under the preset condition of signal and noise, the optimal parameter $a$ found by AFSA is 9792 . If the parameter is used for stochastic resonance processing, the evaluation function of the output signal can reach the maximum value of 8.53 .

To sum up, for the seven different signals, the algorithm reaches the convergence state with the fastest three generations and the slowest six generations, and the optimal system parameters that maximize the evaluation function are obtained, which proves the effectiveness of the algorithm.

Taking 2ASK, BPSK, and 16QAM as examples, the input and output waveforms are compared in Fig. 11, which prove PSR can strengthen the signal and remove the noise efficiently.

\subsection{Experiment 2: Robustness test}

In a real environment, the parameters of the signal are various. Therefore, the 16QAM with high universality, which is often used in satellite communication, will be set as an example with different parameters and repeated tests to verify the robustness of the algorithm. In the process of enlarging the symbol rate from $1 \times 10^{3}$ to $1.6 \times 10^{4} \mathrm{Bd}$, the carrier frequency is set to $4,6,8,10$, and 12 times, respectively, and the sampling rate is uniformly set as $f \mathrm{~s}=100 f \mathrm{c}$. Because the oversampling rate $\left(f_{\mathrm{s}} / R \mathrm{~s}\right)$ varies, the signal length is different under the same number of symbols, so the signal is uniformly intercepted by $1 \times 10^{4}$ points with $\mathrm{SNR}=0 \mathrm{~dB}$. The experiments are repeated 100 times for each parameter, and the parameters of AFSA keep the same as experiment 1 . Finally, the average convergence times were recorded.
The data in Table 1 indicate that the average convergence times remain stable when the symbol rate and carrier frequency change, which can prove two points: first, the convergence speed of the algorithm is not affected by the signal parameters; second, the algorithm can converge to the signals with different parameters and obtain the optimal system parameters adaptively. The robustness of the algorithm under different signal parameters is significant because the target signal is unknown. Experiment 2 shows that the proposed method is robust.

\subsection{Experiment 3: Quality improvement test}

The purpose of this study is to find the optimal stochastic resonance parameters so that the signal quality can be improved. In order to quantitatively measure the signal enhancement effect, we employ the measuring function YSRIF to test the signals under different SNR. Define YI (YSRIF improvement) as follows:

$$
\mathrm{YI}=Y \text { SRIF_out }-Y \text { SRIF_in }
$$

$Y$ SRIF _ in and YSRIF _ out are the measuring functions of the input and output signals, respectively. It is actually a relative signal-to-noise ratio gain measuring method, which can reflect the improvement of signal quality by stochastic resonance processing. In order to clearly present the results, we take 2ASK, BPSK, and 16QAM as examples. Let the SNR of the signals be expanded from -15 to $10 \mathrm{~dB}$ at intervals of $1 \mathrm{~dB}$. The rest of the signal parameters are the same as experiment 1 . Draw the curve of YI as SNR grows as Fig. 12.

In the change of the SNR from low to high, YI is firstly increased and then gradually decreased. The overall values of YI are positive, meaning that the algorithm improves the signal energy to different degrees. When SNR is $-10 \mathrm{~dB}$, YI is most significant. When the SNR is high, the degree of the enhancement is little, which is because the signal energy is already strong and the noise energy is already weak on the contrary. It can be deduced from experiment 3 that stochastic resonance can highlight the excellent performance at low signal-to-noise ratio and is suitable for weak IF signal enhancement.

Table 1 Average convergence times under different signal parameters

\begin{tabular}{llllll}
\hline & $R \mathrm{~s}=$ & $R \mathrm{~s}=$ & $R \mathrm{~s}=$ & $R \mathrm{~s}=$ & $R \mathrm{~s}=$ \\
& $1 \times 10^{3}$ & $2 \times 10^{3}$ & $4 \times 10^{3}$ & $8 \times 10^{3}$ & $1.6 \times 10^{4}$ \\
\hline $\mathrm{fC}_{\mathrm{C}}=4 \mathrm{Rs}$ & 4.5 & 5.1 & 4.6 & 4.8 & 4.7 \\
$\mathrm{fC}_{\mathrm{C}}=6 \mathrm{Rs}$ & 5.4 & 4.3 & 4.7 & 5.4 & 4.5 \\
$\mathrm{fC}_{\mathrm{C}}=8 R \mathrm{Rs}$ & 4.8 & 4.7 & 4.9 & 4.3 & 5.0 \\
$\mathrm{fC}_{\mathrm{C}}=10 R \mathrm{Rs}$ & 5.0 & 4.4 & 4.4 & 4.7 & 5.0 \\
$\mathrm{fC}_{\mathrm{C}}=12 R s$ & 4.6 & 4.3 & 4.8 & 4.8 & 4.9
\end{tabular}




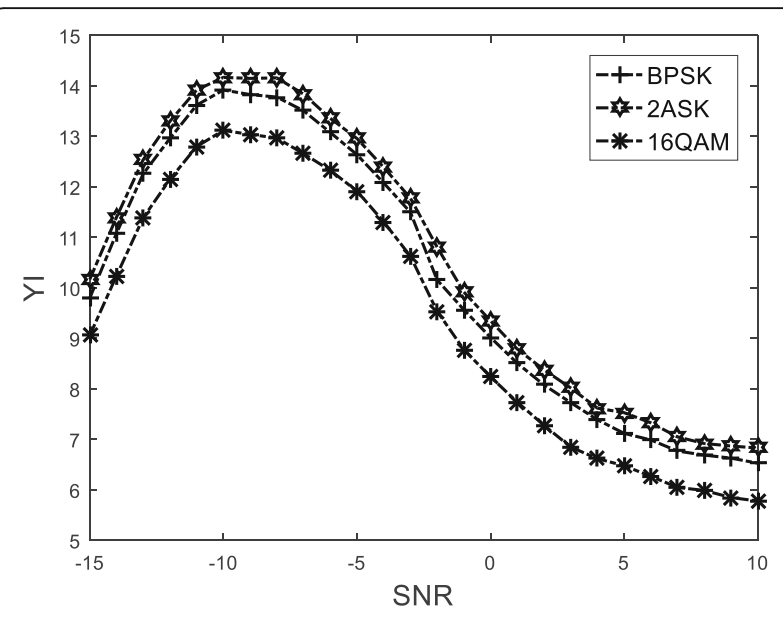

Fig. 12 The change curve of $Y$ I with SNR growth

\subsection{Experiment 4: Contrast test}

The previous three experiments can prove that the proposed method is feasible and applicable from the aspect of algorithm performance. In order to justify it more comprehensively, we need to compare it with existing methods. However, before this study, no one deals IF digital signals with adaptive parameter-tuning stochastic resonance. Therefore, we use the fixed parameter which is set according to the lower limit of the optimization range as the contrast and the optimal parameter obtained from the proposed method, to process the noisy signals respectively and compare them by YI. Take 2ASK, BPSK, and 16QAM as examples and keep the signal parameters as same as experiment 1 except from SNR. The optimal parameter is obtained from the average value after 100 experiments. $\Delta \mathrm{YI}$ is the increasement of YI.

From Table 2, we can see that compared with the stochastic resonance under fixed system parameter, this method can get the optimal system parameter for noisy signals to achieve the best enhancement. For example, we can get the optimum parameter of 5545 for 2ASK with -5 SNR. Compared with the fixed parameter $1000, \Delta \mathrm{YI}$ can reach positive $8.2 \mathrm{~dB}$, which means the enhancement effect of the weak signal reaches the highest level under the optimum parameter.

\section{Conclusions}

An adaptive parameter-tuning stochastic resonance method for three types of weak IF digital signals (MASK, MPSK, and MQAM) is proposed, and a frame of signal enhancement preprocessing is presented here. The method is capable of adaptively adjusting the SR system parameters in accordance with different noisy signals, enhancing the characteristics and improving the quality of signals by AFSA. According to the theoretical analysis and simulation experiments, the algorithm is proven effective. It has the following advantages: First, the method of SVD with fixed order is employed to build the evaluation function of the output signal, effectively measuring the aperiodic signals from non-cooperative recipient. Second, the effects of different system parameters on the signal are investigated, and the optimization parameters are reduced by half, significantly increasing the efficiency of calculation. Third, the algorithm does not require accurate prior knowledge and can adaptively deal with the large-parameter digital communication signals in non-cooperative environment, which is of practical importance. Fourth, the application of stochastic resonance is expanded, and some innovative ideas for signal processing are provided.

In the meantime, there are undoubtedly many other types of signals in digital communication. The subsequent step will focus on expanding the applicable signal types to make it a more complete receiver architecture.

Table 2 Contrast experiment results

\begin{tabular}{|c|c|c|c|c|c|c|}
\hline \multirow[t]{2}{*}{ Signal } & \multirow{2}{*}{$\begin{array}{l}\text { SNR } \\
\text { (dB) }\end{array}$} & \multicolumn{2}{|c|}{ Traditional method } & \multicolumn{2}{|l|}{ Proposed method } & \multirow{2}{*}{$\begin{array}{l}\Delta \mathrm{YY} \\
(\mathrm{dB})\end{array}$} \\
\hline & & Fixed parameter & $\mathrm{YI}(\mathrm{dB})$ & Optimal parameter & $\mathrm{YI}(\mathrm{dB})$ & \\
\hline \multirow[t]{3}{*}{ 2ASK } & -5 & 1000 & 5.44 & 5545 & 13.64 & +8.2 \\
\hline & -10 & & 7.26 & 6976 & 15.13 & +7.87 \\
\hline & -15 & & 3.37 & 6037 & 10.55 & +7.18 \\
\hline \multirow[t]{3}{*}{ BPSK } & -5 & 1000 & 5.03 & 8329 & 13.34 & +8.31 \\
\hline & -10 & & 6.98 & 8003 & 14.65 & +7.67 \\
\hline & -15 & & 4.86 & 8536 & 10.68 & +5.82 \\
\hline \multirow[t]{3}{*}{ 16QAM } & -5 & 1000 & 5.03 & 5067 & 12.53 & +7.5 \\
\hline & -10 & & 6.64 & 6543 & 14.36 & +7.72 \\
\hline & -15 & & 3.94 & 5500 & 10.44 & +6.5 \\
\hline
\end{tabular}




\section{Abbreviations}

AFSA: Artificial fish swarm algorithm; ASR: Aperiodic stochastic resonance; IF: Intermediate frequency; MASK: Multiple amplitude shift keying; MDL: Minimum description distance; MPSK: Multiple phase shift keying; MQAM: Multiple quadrature amplitude modulation; PAM: Pulse amplitude modulation; PSR: Parameter-tuning stochastic resonance; RF: Radio frequency; SNR: Signal-noise ratio; SR: Stochastic resonance; SVD: Singular value decomposition

\section{Acknowledgements}

The authors would like to thank IEU for this support and anyone who support this paper to be published.

\section{Funding}

This work has been funded by the National Natural Science Foundation of China, under the project No.61401511.

\section{Authors' contributions}

ZZ finished the experiments and write parts of the manuscript. JM finished the theory part. Both authors read and approved the final manuscript.

\section{Consent for publication}

$$
\text { Not applicable. }
$$

\section{Competing interests}

The authors declare that they have no competing interest

\section{Publisher's Note}

Springer Nature remains neutral with regard to jurisdictional claims in published maps and institutional affiliations.

\section{Received: 28 December 2018 Accepted: 15 March 2019}

\section{Published online: 11 April 2019}

\section{References}

1. C.L. Nikias, J.M. Mendel, Signal processing with higher-order spectra. IEEE Signal Process. Mag. 10(3), 10-37 (1993)

2. Z. Zhang, W. Yang, Q. Ding, in Proceedings of the IEEE Fourth International Conference on Natural Computation. Communication signal processing at low SNR using higher-order spectrum (IEEE Press, Jinan, 2008), pp. 65-69

3. J. Zhang, X. Chen, K.P. Peppas, et al., On high-order capacity statistics of Spectrum aggregation systems over shadowed fading channels. IEEE Trans. Commun. 65(2), 935-944 (2017)

4. Z. Huo, Y. Zhang, P. Francq, et al., Incipient fault diagnosis of roller bearing using optimized wavelet transform based multi-speed vibration signatures. IEEE Access 5, 19442-19456 (2017)

5. J.S. Wheatley, J.W. loup, G.E. loup, Wavelet detection and denoising of lowfrequency chirp signals. J. Acoust. Soc. Am. 109(5), 2296 (2001)

6. Z. Xing, Y. Gao, Method to reduce the signal-to-noise ratio required for modulation recognition based on logarithmic properties. IET Commun. 12(11), 1360-1366 (2018)

7. W.A. Gardner, C.M. Spooner, in Proceedings of the 21st Century Military Communications-What's Possible? Conference Record. Cyclic spectral analysis for signal detection and modulation recognition (IEEE Press, San Diego, 1988), pp. 419-424

8. H. Sun, S. Yuan, Y. Luo, Cyclic spectral analysis of vibration signals for centrifugal pump fault characterization. IEEE Sensors J. 18(7), 2925-2933 (2018)

9. R. Benzi, A. Sutera, A. Vulpiani, Mechanism of stochastic resonance. J. Phys. A: Gen. Phys. 14(11), 453 (2004)

10. J.J. Collins et al., Aperiodic stochastic resonance. Phys. Rev. E 54(5), 5575-5584 (1996)

11. B. Xu et al., Stochastic resonance with tuning system parameters: The application of bistable systems in signal processing. Chaos, Solitons Fractals 13(4), 633-644 (2002)

12. M. Singh, A. Verma, N. Sharma, Optimized multi-stable stochastic resonance for the enhancement of pituitary microadenoma in MRI. IEEE J. Biomed. Health Inform. 22(3), 862-873 (2017)

13. R. Kihara, Y. Yamanashi, N. Yoshikawa, Power reduction of Josephson random access memory using stochastic resonance. IEEE Trans. Appl. Supercond. 26(8), 1-4 (2016)
14. H. Calisto, F.R. Humire, Stochastic resonance in simple electrical circuits driven by quadratic Gaussian noise. IEEE Trans. Circuits Syst. Express Briefs 64(8), 937-941 (2017)

15. G. Zhang et al., Stochastic resonance in second-order underdamped system with exponential bistable potential for bearing fault diagnosis. IEEE Access 6, 42431-42444 (2018)

16. S. Lu et al., Online fault diagnosis of motor bearing via stochasticresonance-based adaptive filter in an embedded system. IEEE Trans. Syst. Man Cybern. Syst. Hum. 47(7), 1111-1122 (2017)

17. X. Huang et al., Realising the decomposition of a multi-frequency signal under the coloured noise background by the adaptive stochastic resonance in the non-linear system with periodic potential. IET Signal Proc. 12(7), 930-936 (2018)

18. F. Duan, D. Abbott, Binary modulated signal detection in a bistable receiver with stochastic resonance. Physica A Stat Mechanics Appl 371(1), 173-190 (2007)

19. S. Sun, B. Lei, On an aperiodic stochastic resonance signal processor and its application in digital watermarking. Signal Process. 88(8), 2085-2094 (2008)

20. S. Dalabaev et al., in Proceedings of 2011 Cross Strait Quad-Regional Radio Science and Wireless Technology Conference. The application of the system of parameter tuning stochastic resonance in baseband signal processing (IEEE Press, Harbin, 2011), pp. 1387-1389

21. J. Liu et al., A novel parameter-tuned stochastic resonator for binary PAM signal processing at low SNR. IEEE Commun. Lett. 18(3), 427-430 (2014)

22. L. Liang et al., Comparison results of stochastic resonance effects realised by coherent and non-coherent receivers under Gaussian noise. IET Commun. 11(8), 1180-1186 (2017)

23. Y. Zhan, C. Duan, in Proceedings of IEEE International Conference on Communication Software and Networks. The application of stochastic resonance in parameter estimation for PSK signals (IEEE Press, Chengdu, 2015), pp. 166-172

24. L. Tong et al., A PSO optimization scale-transformation stochasticresonance algorithm with stability mutation operator. IEEE Access 99, 1167-1176 (2018)

25. G. Zhou et al., Artificial fish swarm based power allocation algorithm for MIMO-OFDM relay underwater acoustic communication. IET Commun. 12(9), 1079-1085 (2018)

26. M. Neshat et al., Artificial fish swarm algorithm: a survey of the state-of-theart, hybridization, combinatorial and indicative applications. Artif. Intell. Rev. 42(4), 965-997 (2014)

27. D.Y. Kong, H. Peng, J.Q. Ma, Adaptive stochastic resonance method based on artificial-fish swarm optimization. Acta Electron. Sin. 45(8), 1864-1872 (2017)

28. W. Zhu, L. Min, Weak signal intelligent detection system based on stochastic resonance and artificial fish swarm algorithm. Chin. J. Sci. Instrum. 34(11), 2464-2470 (2013)

29. Chen et al., Theory of the stochastic resonance effect in signal detection-part II: variable detectors. IEEE Trans. Signal Process. 56(10), 5031-5041 (2008)

30. N. Mc, K. Wiesenfeld, R. Roy, Theory of stochastic resonance. Phys. Rev. A 39(9), 4854-4869 (1989)

31. N. Mc, K. Wiesenfeld, R. Roy, Observation of stochastic resonance in a ring laser. Phys. Rev. Lett. 60(25), 2626-2629 (1988)

32. L. Gammaitoni et al., Stochastic resonance. Rev. Mod. Phys. 70(1), 45-105 (2008)

33. D.X. Yang, The analysis of stochastic resonance of periodic signal with large parameters. Acta Phys. Sin. 61(8), 963-963 (2012)

34. J.J. Collins, C.C. Chow, T.T. Imhoff, A periodic stochastic resonance in excitable systems. Phys. Rev. E 52(4), 3321-3323 (1995)

35. F. Duan, B. Xu, Parameter-induced stochastic resonance and baseband binary, pam signals transmission over an awgn channel. Int. J. Bifurcation Chaos 13(2), 411-425 (2003)

36. A. ljaz, A.B. Awoseyila, B.G. Evans, Signal-to-noise ratio estimation algorithm for advanced DVB-RCS systems. IEEE Trans. Broadcast. 58(4), 603-608 (2012)

37. M. Andersin, N.B. Mandayam, R.D. Yates, Subspace based estimation of the signal to interference ratio for TDMA cellular systems. Wirel. Netw 4(3), 241-247 (1998) 